\title{
Colours of intermediate $z$ bulges in Groth and GOODS-N
}

\author{
Lilian Domínguez-Palmero ${ }^{1} \dagger$ and Marc Balcells ${ }^{1}$ \\ ${ }^{1}$ Instituto de Astrofísica de Canarias, C/ Vía Láctea s/n, 38200 La Laguna, Tenerife, Spain \\ email: ldp@iac.es balcells@iac.es
}

\begin{abstract}
The chronology of bulge and disk formation is a major unsolved issue in galaxy formation, which impacts on our global understanding of the Hubble sequence. We present colours of the nuclear regions of intermediate-redshift disk galaxies, with the aim of obtaining empirical information of ages of bulges at intermediate $z$. We work with a sample of 248 galaxies (123 inclined + 125 face-on) from the HST Groth Strip Survey (Groth et al. 1994) and another one with 404 objects (214 inclined +190 face-on) from the HST GOODS-N field (Giavalisco et al. 2004), covering redshifts $0.1<z<1.3$. Those samples are apparent-diameter limited at $R>1.4^{\prime \prime}$. We find that, as in the Local Universe, the minor axis color profiles are negative (bluer outward), and fairly gentle, indicating that bulge colours are not distinctly different from disk colours. We apply a conservative criterion to identify bulges and potential precursors of present-day bulges, based on nuclear excess surface brightness above the exponential profile of the outer parts. For galaxies with central brightness excesses, rest-frame colour distributions show a red sequence that confirms the finding of very red bulges by Koo et al. (2005), using independent methods. In contrast, galaxies without central brightness excesses show typical colours of star-forming populations. Clearly, something had truncated star formation in many high-density cores, already at $z \sim 1$. The truncation epoch is uncertain, $1.5<z<10$. The colour-magnitude distribution of intermediate- $z$ bulges shows more colour dispersion than that of bulges in the Local Universe. About 50\% of bulges are as red as local bulges, while the remainder are significantly bluer, a possible sign of late bulge formation. We also find that bulge colours correlate with integrated galaxy colours and with their disk colours.
\end{abstract}

Keywords. galaxies: bulges, galaxies: fundamental parameters, galaxies: stellar content

\section{Selection of galaxies with measurable bulge colours and central colour measurements}

Selecting intermediate-redshift bulges for colour measurements is difficult. Their small apparent sizes and the irregular morphologies of many disk galaxies at those redshifts hamper classical bulge-disk decomposition. Besides, ideally we wish to include not only bona-fide bulges, but also likely precursors of present-day bulges. We adopt a simple surface brightness criterion to measure the prominence of the bulges above the disks: we define the bulge prominence as the excess central brightness above the inward extrapolation of an exponential fit to the outer major axis profile, measured in the most infrared band. We include in our bulge sample all those galaxies with prominence brighter than 1 mag. Our selection ensures that all the selected galaxies have a well defined, extended bulge in which we can measure colours. Galaxies in the no-bulge group either have no bulges, or have an unresolved bulge. We estimated bulge colors from the color profiles derived on wedge-shaped apertures opening on the clean semiminor axes of the galaxies at $0.85 \mathrm{kpc}$ from the centre.

† C/ Vía Láctea s/n, 38200 La Laguna, Tenerife, Spain 

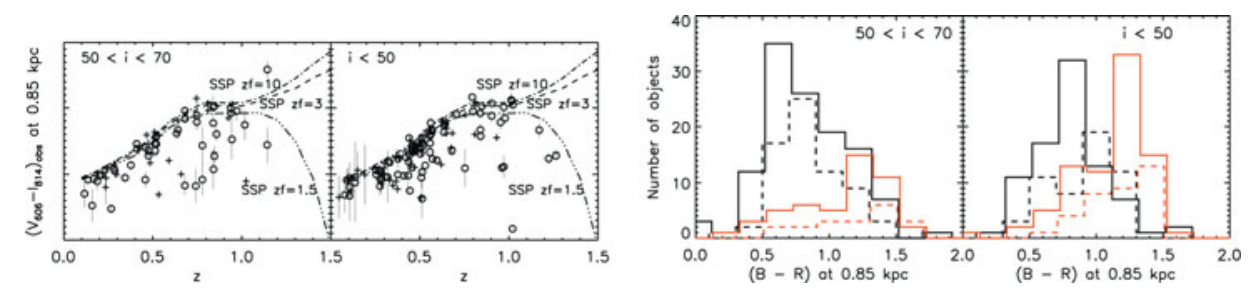

Figure 1. (a) Observed-frame nuclear $(F 606 W-F 814 W)$ colours for galaxies with bulges vs $z$. Groth bulges: crosses; GOODS-N bulges: black open circles. Left: inclined sample, right: face-on sample. Lines: models of passive evolution populations (SSP with solar metallicity, Chabrier $2003 \mathrm{IMF}$, no dust) with $z_{\text {form }}=10,3,1.5$. (b) Histograms for rest-frame $(B-R)$ colour; red (or grey) lines: colour distribution of bulges; black lines: nuclear colour distribution of galaxies without bulges. Solid lines: GOODS-N sample; dashed lines: Groth sample.
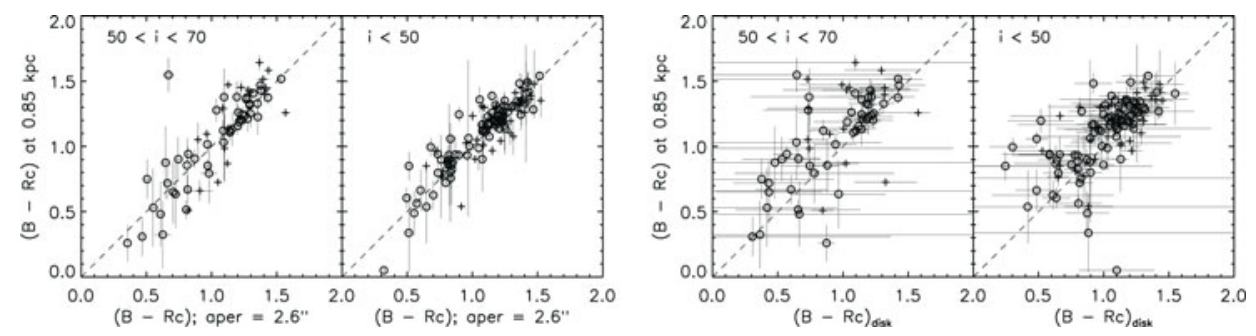

Figure 2. (a) Rest-frame $(B-R)$ colours of bulges vs rest-frame integrated galaxy $(B-R)$ colour. Groth bulges: crosses; GOODS-N bulges: black open circles. Left: inclined sample, right: face-on sample. (b) Rest-frame $(B-R)$ colours vs rest-frame disk $(B-R)$ colour. Groth bulges: crosses; GOODS-N bulges: black open circles. Left: inclined sample, right: face-on sample.

\section{Results}

Observed colours of some bulges form a red envelope (see Figure 1(a)) that can fit well models of passive evolution populations with formation redshifts that range from 10 to 1.5. This result would imply a big uncertainty in the age of red bulges, the only thing we can assure is that they are not forming stars at this moment. We also find a wide dispersion of bulges toward bluer colours. In the colour histograms (see Figure 1(b)) we see that bulge samples are dominantely redder than the non-bulge samples, in all cases; recalling that bulges are defined here on the basis of central brightness excess only, this result indicates an association of red colors and high central surface brightness excess, extending to redshifts $z \sim 0.9$.

When comparing nuclear colours and global colours, in Figure 2(a), we see that they are very similar to each other. This result was found for $z=0$ galaxies by Peletier and Balcells (1996) and is here extended up to $z=0.9$. Moreover, bulge colours correlate with disk colours (see Figure 2(b)), galaxies with redder bulges have redder disks: in many cases the colour differences between bulges and disks is smaller than that between bulges of different galaxies.

\section{References}

Peletier, R. F. \& Balcells, M. 1996, AJ 111, 2238

Koo, D. C. et al. 2005, ApJ 157, 175-217

Groth, E. J. et al. 1994, BAAS 185, 5309

Giavalisco, M. et al. 2004, ApJ 600, L93-L98 